\title{
Striving for decreased post-transplant hepatocellular carcinoma recurrence without excluding potentially curable patients: the utility of tumor biology
}

\author{
Russell Evan Rosenblatt, Karim Jarir Halazun \\ Weill Cornell Medicine, New York, USA \\ Correspondence to: Karim Jarir Halazun. Weill Cornell Medicine, 525 East 68th Street, New York, USA. Email: kah7007@med.cornell.edu. \\ Comment on: Grąt M, Stypułkowski J, Morawski M, et al. Shadows Behind Using Simple Risk Models in Selection of Hepatocellular Carcinoma \\ Patients for Liver Transplantation. Ann Surg 2018. [Epub ahead of print].
}

Submitted Mar 27, 2019. Accepted for publication Apr 10, 2019.

doi: 10.21037/hbsn.2019.04.09

View this article at: http://dx.doi.org/10.21037/hbsn.2019.04.09

The Milan criteria truly transformed the field of transplantation for hepatocellular carcinoma by demonstrating that excellent recurrence-free survival (RFS) can be achieved in small tumors (1). Over the years, the allowable tumor size and number prior to transplantation was challenged by several groups and thus continued to expand, but the use of simple morpho-metrics always limited the ability to predict tumor recurrence, as markers of tumor biology were not routinely used. More recently, multiple studies have combined biomarkers with tumor size and number to create increasingly accurate scoring systems to better predict RFS. The adoption of the AFP model in France (2), accounting for log AFP and tumor size and number, in addition to the newest model by Mazzaferro et al., Metroticket 2.0 (3), a more fluid model utilizing similar criteria to the AFP model, join the MORAL score (4), which incorporates the neutrophil lymphocyte ratio (NLR), and the NYCA score (5), which incorporates AFP response, as the best current predictors of RFS available to date.

It is in this setting that Grat et al. (6) determined to reassess whether newer scoring systems, in this case the AFP model and the Metroticket 2.0, were actually superior to the gold standard still utilized in most of the world, the Milan criteria. Using a retrospective cohort of 282 patients, the study demonstrated that despite the AFP model and the Metroticket 2.0 predicting RFS more accurately, there was no net reclassification improvement over the Milan criteria. The authors note that patients outside of Milan criteria but still within the AFP model and Metroticket 2.0 had worse 5 -year RFS than those within Milan criteria (74\% vs. $75 \%$ vs. $87 \%$, respectively). Therefore, the authors conclude that newer scoring systems, such as the AFP model and Metroticket 2.0, allow for the inclusion of patients at higher risk for post-OLT recurrence.

While the authors note a limitation of selection bias due to inclusion of nearly one-fifth of the sample with 4 tumors or more, there are other biases within the sample itself. Firstly, the authors state that selection of HCC patients for OLT in their institution seems non-standardized and follows "UCSF or Up-To-7 Criteria and be expanded based on tumor board recommendations". With this in mind, it seems curious that $<50 \%$ of the patients in the study received locoregional as downstaging therapy or as a bridge to transplant, currently the standard of treating these patients. Secondly, as the authors point out, they chose to censor deaths in the survival analysis rather that account for death as a competing risk to recurrence. This has been recently shown to be a superior way to predict actual cumulative hazards of recurrence by at least 2 HCC models (one of which is being studied here) (5), as patients who die prior to recurrence can never recur and therefore should not be censored. The recurrences in the paper are therefore underestimated and may cause Metroticket 2.0 to underperform. Thirdly, the 282 patient samples are markedly smaller than the cohorts utilized to design or validating the AFP model and Metroticket 2.0. This study, therefore, has limited external validity, especially when compared to the international cohorts designed to validate the AFP model (7) and Metroticket 2.0 (3). Lastly, the reported "doubled" recurrence rates of patients beyond the Milan criteria but 
within the AFP and Metroticket 2.0 are not evident by the data presented. In fact, both models predicted recurrences well compared to Milan in this dataset, approaching $75 \%$ at five years for their high-risk groups.

The progression from morphologic-based selection criteria to the inclusion of biomarkers represents a marked improvement in predicting RFS, but vital information, namely the ability to account for changes in tumor aggression as measured by changes in AFP, is still lacking. Both the AFP models and Metroticket model are effective pre-operative selection tools, but, they incorporate a static AFP value. Previous studies however have demonstrated that AFP downstaging resulted in markedly improved survival (8). For this reason, NYCA, with its inclusion of the response of AFP, from listing to time of transplant, in lieu of simple AFP at time of listing, symbolizes the next major shift in predicting RFS. The AFP response was crucial in identifying a subgroup of patients who normally would not be considered for OLT, those with AFP $>1,000 \mathrm{ng} / \mathrm{mL}$, who can respond to treatment have similar outcomes to those from $200-1,000 \mathrm{ng} / \mathrm{mL}$ who drop $<200 \mathrm{ng} / \mathrm{mL}$. Metroticket 2.0 and the French AFP model can also theoretically both be recalculated once patients are down-staged and scores reassessed, thus reconsidering listing status. A look at these outcomes would be of interest.

Similar to how the Milan criteria changed the fundamental approach to OLT in patients with HCC, the newer scoring systems have pushed the boundaries again. These scoring systems, by accounting for tumor biology, have shown that over 70\% 5-year RFS survival (and therefore potential cure) is achievable in patients outside of the Milan criteria. When most OLT centers determine candidacy based on 5 -year overall survival $>50 \%$, further excluding patients who easily surpass this expectation - those who fall outside of the Milan criteria but within the AFP model or Metroticket 2.0 in this study-seems unjust. Given the results, of the study by Grat et al. (6) and those of other scoring systems including biomarkers and morphologic characteristics (2-5), the approach to OLT candidacy for patients with HCC should not be exclusive, but rather more inclusive, allowing patients with larger or more numerous tumors with good biological behavior to proceed to OLT, expanding the recipient pool for patients with HCC.

\section{Acknowledgments}

None.

\section{Footnote}

Conflicts of Interest: The authors have no conflicts of interest to declare.

\section{References}

1. Mazzaferro V, Regalia E, Doci R, et al. Liver transplantation for the treatment of small hepatocellular carcinomas in patients with cirrhosis. $\mathrm{N}$ Engl J Med 1996;334:693-9.

2. Duvoux C, Roudot-Thoraval F, Decaens T, et al. Liver transplantation for hepatocellular carcinoma: A model including $\alpha$-fetoprotein improves the performance of milan criteria. Gastroenterology 2012;143:986-94.e3; quiz e14-5.

3. Mazzaferro V, Sposito C, Zhou J, et al. Metroticket 2.0 Model for Analysis of Competing Risks of Death After Liver Transplantation for Hepatocellular Carcinoma. Gastroenterology 2018;154:128-39.

4. Halazun KJ, Najjar M, Abdelmessih RM, et al. Recurrence after liver transplantation for hepatocellular carcinoma. Ann Surg 2017;265:557-64.

5. Halazun KJ, Tabrizian P, Najjar M, et al. Is it Time to Abandon the Milan Criteria?: Results of a Bicoastal US Collaboration to Redefine Hepatocellular Carcinoma Liver Transplantation Selection Policies. Ann Surg 2018;268:690-9.

6. Grat M, Stypułkowski J, Morawski M, et al. Shadows Behind Using Simple Risk Models in Selection of Hepatocellular Carcinoma Patients for Liver Transplantation. Ann Surg 2018. [Epub ahead of print]

7. Piñero F, Tisi Baña M, de Ataide EC, et al. Liver transplantation for hepatocellular carcinoma: evaluation of the alpha-fetoprotein model in a multicenter cohort from Latin America. Liver Int 2016;36:1657-67.

8. Merani S, Majno P, Kneteman NM, et al. The impact of waiting list alpha-fetoprotein changes on the outcome of liver transplant for hepatocellular carcinoma. J Hepatol 2011;55:814-9.

Cite this article as: Rosenblatt RE, Halazun KJ. Striving for decreased post-transplant hepatocellular carcinoma recurrence without excluding potentially curable patients: the utility of tumor biology. HepatoBiliary Surg Nutr 2019;8(5):541-542. doi: 10.21037/hbsn.2019.04.09 